\title{
Conditions Where the Chaotic Set Has a Non-Empty Residual Julia Set for Two Classes of Meromorphic Functions*
}

\author{
Patricia Domínguez, Iván Hernández \\ Facultad de Ciencias Fsico-Matemáticas, Benemérita Universidad Autónoma de Puebla, Puebla, Mexico \\ Email: pdsoto@fcfm.buap.mx, ivanho_5@hotmail.com
}

Received August 15, 2013; revised September 15, 2013; accepted September 23, 2013

Copyright (c) 2013 Patricia Domínguez, Iván Hernández. This is an open access article distributed under the Creative Commons Attribution License, which permits unrestricted use, distribution, and reproduction in any medium, provided the original work is properly cited.

\begin{abstract}
We define the Fatou and Julia sets for two classes of meromorphic functions. The Julia set is the chaotic set where the fractals appear. The chaotic set can have points and components which are buried. The set of these points and components is called the residual Julia set, denoted by $J_{r}(f)$, and is defined to be the subset of those points of the Julia set, chaotic set, which do not belong to the boundary of any component of the Fatou set (stable set). The points of $J_{r}(f)$ are called buried points and the components of $J_{r}(f)$ are called buried components. In this paper we extend some results related with the residual Julia set of transcendental meromorphic functions to functions which are meromorphic outside a compact countable set of essential singularities. We give some conditions where $J_{r}(f) \neq \varnothing$.
\end{abstract}

Keywords: Fatou Set; Julia Set; Residual Julia Set; Buried Points; Buried Components

\section{Introduction}

Let $X, Y$ be Riemann surfaces (complex 1-manifolds) and $D_{f}$ be an arbitrary non-empty open subset of $X$. We define

$$
\begin{aligned}
& \operatorname{Hol}(X, Y)=\left\{f: D_{f} \rightarrow Y \mid f \text { is analytic }\right\} \\
& \text { and } \operatorname{Hol}(X, X)=\operatorname{Hol}(X) .
\end{aligned}
$$

The set of singular values of $f \in \operatorname{Hol}(X, Y)$ is $S V(f)=\overline{C(f) \cup A(f)}$, where $C(f)$ is the set of critical values and $A(f)$ is the set of asymptotic values.

Let $f \in \operatorname{Hol}(X)$, the sequence formed by its iterates will be defined and denoted by $f^{0}:=\mathrm{Id}, f^{n}:=f \circ f^{n-1}$, $n \in \mathbb{N}$. The study makes sense and is non-trivial when $X$ is either the Riemann sphere $\hat{\mathbb{C}}$, the complex plane $\mathbb{C}$ or the complex plane minus one point, this is $\mathbb{C} \backslash\{0\}$.

Taking $X=\mathbb{C}$ and $Y=\hat{\mathbb{C}}$ we deal with the following classes of meromorphic maps.

$$
\begin{gathered}
\mathcal{M}=\{f: X \rightarrow Y \mid f \text { is transcendental meromorphic with at least one not omitted pole }\} . \\
\mathcal{K}=\{f: Y \backslash B \rightarrow Y \mid B \text { is a compact countable set and } f \text { is meromorphic }\} .
\end{gathered}
$$

The set $B$ is formed by the essential singularities of $f$, where $f$ is non-constant. We assume $B$ to have at least two elements and $f$ to have poles. With this assumption we have $\mathcal{M} \cap \mathcal{K}=\varnothing$.

If $f$ is a map in any of the classes above the Fatou set

*The authors were supported by CONACYT grant 128005.
$F(f)$ consists of all points $z \in X$ (or $z \in Y \backslash B$ ) such that the sequence of iterates of $f$ is well defined and forms a normal family in a neighbourhood of $z$. The Julia set is the complement of the Fatou set, denoted by $J(f)=(F(f))^{c}$. The Fatou and the Julia sets are also known as the stable and the chaotic sets respectively. In 
the Julia set or chaotic set is easy to find fractals, examples of this fact are below. The fractals are typically selfsimilar patterns, where self-similar means they are "the same from near as from far" [1].

Examples of functions in class $\mathcal{M}$ live in the family $f_{\lambda, \mu}=\lambda \mathrm{e}^{z}+\frac{\mu}{z}$ studied in [2]. The stable set (Fatou set) and the chaotic set (Julia set) for the parameters $\lambda=-4$, $\mu=-1$ can be seen in Figure 1.

Examples of functions in class $\mathcal{K}$ can be found in the family $f_{c}=R(z) \mathrm{e}^{\frac{\epsilon}{z^{2}-c}}$, where $R(z)$ is a rational function, $\epsilon \in \mathbb{R}, \epsilon \neq 0$ and $c \in \mathbb{C}$. We do not have any picture of the Fatou and Julia set but the Julia set should be a fractal for some parameters $c$ and $\epsilon$ sufficiently small.

Class $\mathcal{M}$ was initially studied by Baker, Kotus and Yi Nian [3-6]. The class $\mathcal{K}$ has been introduced and studied by Bolsch in [7-9].

Many properties of $J(f)$ and $F(f)$ are much the same for all classes above but different proofs are needed and some discrepancies arise. For functions in classes $\mathcal{M}$ or $\mathcal{K}$ we recall some properties of the Fatou and Julia sets: the Fatou set $F(f)$ is open and the Julia set $J(f)$ is closed; the Julia set is perfect and non-empty; the sets $J(f)$ and $F(f)$ are completely invariant under $f$; and finally the repelling periodic points are dense in $J(f)$.

A Fatou component for a function in class $\mathcal{M}$ or $\mathcal{K}$ can be periodic, pre-periodic or wandering. The possible dynamics of a periodic component of the Fatou set is either attracting, parabolic, Siegel disc, Herman ring or Baker domain. Figure 1 is an example of a Baker domain for the function $f_{-4,-1}=-4 \mathrm{e}^{z}+\frac{-1}{z}$, see [2] for details.

It was proved in [5], for functions in class $\mathcal{M}$, and in [8], for functions in class $\mathcal{K}$, that a periodic Fatou component (of arbitrary period) is simply, doubly or infinitely connected.

In [6] the authors proved that for functions in class

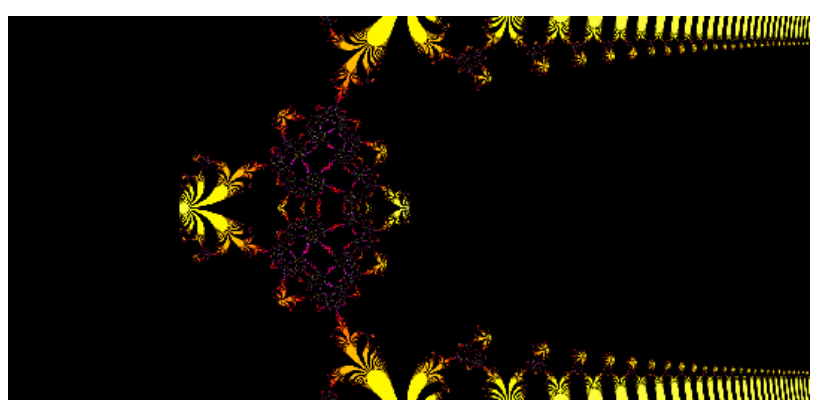

Figure 1. The chaotic set, which is a fractal, with colors and the Fatou set on black.
$\mathcal{M}$ with a finite set of singular values there are neither wandering components nor Baker domains. The same statement works for functions in class $\mathcal{K}$ and the proofs are similar to those in [6].

We define the residual Julia set of $f$ denoted by $J_{r}(f)$ as the set of those points of $J(f)$ which do not belong to the boundary of any component of the Fatou set $F(f)$. The points of $J_{r}(f)$ are called buried points and the components of $J_{r}(f)$ are called buried components. This is the Residual Julia set (buried points and buried components) which are in the chaotic set.

This concept was first introduced in the context of Kleinian groups by Abikoff in [10,11]. In [12], McMullen defined a buried component of a rational function to be a component of the Julia set which does not meet the boundary of any component of the Fatou set. Similarly, for a buried point of the Julia set. McMullen gave an example of a rational function with buried components.

Baker and Domínguez in [13] extended some results of Qiao [14] (for rational functions) to have buried points or buried components to functions in class $\mathcal{M}$. In Section 2 we prove that the same results can be extended to functions in class $\mathcal{K}$.

Finally, Section 3 contains Theorems A and B which assure with some conditions that the residual Julia set is not empty for functions in classes $\mathcal{M}$ and $\mathcal{K}$.

\section{Basic Results of the Residual Julia Set for Functions in Classes $\mathcal{M}$ and $\mathcal{K}$}

In this Section we will state some basic results about the residual Julia set which hold for functions in classes $\mathcal{M}$ and $\mathcal{K}$. The proofs of the these results can be found in [13] and [15].

Proposition 2.1. Let $f$ be in class $\mathcal{M}$ or $\mathcal{K}$. If the Fatou set off has a completely invariant component, then the residual Julia set is empty.

Proposition 2.2. Let $f$ be in class $\mathcal{M}$ or $\mathcal{K}$. If there exists a buried component of $J(f)$, then $J(f)$ is disconnected.

Proposition 2.3. Let $f$ be in class $\mathcal{M}$ or $\mathcal{K}$. If $J_{r}(f) \neq \varnothing$, then $J_{r}(f)$ is completely invariant, dense in $J(f)$ and uncountably infinite.

Proposition 2.4. If $f \in \mathcal{K}$ has no wandering domains and $J_{r}(f)=\varnothing$, then there is a periodic Fatou component $U$ such that $J(f)=\partial U$.

\section{Some Conditions When $J_{r}(f) \neq \varnothing$ for Functions in Class $\mathcal{K}$}

In this section we will extend some results related with the residual Julia set for functions in class $\mathcal{M}$ to functions in class $\mathcal{K}$. Qiao in [14] proved the following theorem for rational functions.

Theorem 3.1. Let $f$ be a rational function and 
$J(f) \neq \hat{\mathbb{C}}$. The Julia set $J(f)$ contains buried components if and only if i) $J(f)$ is disconnected and ii) $F(f)$ has no completely invariant component.

Baker and Domínguez in [13] gave the following result which was step towards a generalisation of Theorem 3.1 for functions in class $\mathcal{M}$.

Theorem 3.2. Let $f$ be a meromorphic function in $\mathbb{C}$ with no wandering domains. Assume that the $J(f)$ is not connected and that $F(f)$ has no completely invariant component. Then the residual Julia set $J_{r}(f)$ is non-empty.

If we removed the hypothesis of no wandering domains of Theorem 3.2 and extend it to functions in class $\mathcal{K}$ the statement is as follows.

Theorem A. Let f be a function in class $\mathcal{K}$. If $J(f)$ is not connected and $F(f)$ has no completely invariant component. Then the residual Julia set $J_{r}(f)$ is nonempty, this is $J_{r}(f) \neq \varnothing$.

In order to prove Theorem A we need to state some results for functions in classes $\mathcal{M}$ and $\mathcal{K}$. The following lemma was given in [16] for functions in class $\mathcal{M}$, since the proof works for functions in class $\mathcal{K}$ we do not write it.

Lemma 3.3. If $f \in \mathcal{M}$ or $\mathcal{K}$ and $U$ is a multiply connected periodic Fatou component such that $\partial U=J(f)$, then $U$ is completely invariant.

The following result was given in [17] for functions in class $\mathcal{K}$.

Theorem 3.4. Let $f \in \mathcal{K}$. Suppose that the Fatou set has no completely invariant domain and the Julia set is disconnected in such a way that the Fatou set has a component $H$ of connectivity at least five. Then singleton components are dense and buried in $J(f)$.

\section{Proof of Theorem A.}

If $U$ is a component of the Fatou set, then it can be either periodic, preperiodic or wandering. We will split the proof in two cases the no wandering case and the wandering case.

\section{No wandering case.}

Let $f \in \mathcal{K}$. Assume that there are not wandering domains in the Fatou set and that $J_{r}(f) \neq \varnothing$. By Proposition 2.4 there is a periodic Fatou component $U$ such that $\partial U=J(f)$. The component $U$ is multiply connected since the Julia set, by hypothesis, is not connected. By Lemma 3.3 the component $U$ must be completely invariant which gives us a contradiction. Therefore, the residual Julia set is not empty.

\section{Wandering case.}

We assume that the Fatou set has wandering components. We prove the result in two cases: 1) $f$ has only finite connected Fatou components and 2) $f$ has at least one infinitely connected Fatou component.

1) Since the Julia set is disconnected it consists of uncountable many components. Now as the connectivity of each component of the Fatou set of $f$ is finite, then the number of the boundary components of all Fatou components is countable. Thus the Julia set has uncountably many buried components. Therefore, $J_{r}(f) \neq \varnothing$.

2) If we take $U$ a multiply-connected Fatou component of connectivity $n, n \geq 5, n \in \mathbb{N}$, then the proof follows as the proof of Theorem 3.4 in [17]. Thus singleton buried components are dense in the Julia set. Therefore, $J_{r}(f) \neq \varnothing$.

The following theorem is an extension of Proposition 6.1 given in [16], since the proof given in [16] extends easily to our case, functions in class $\mathcal{K}$, we shall give just a sketch of it.

Theorem B. Let $f \in \mathcal{K}$, and $A \subsetneq(\hat{\mathbb{C}} \backslash B)$ a closed set with non-empty interior. Suppose the following two conditions are satisfied:

- $((\hat{\mathbb{C}} \backslash B) \backslash A) \cap J(f) \neq \varnothing$.

- All the Fatou components of feventually iterate inside $A$ and never leave again. That is, if $\Omega$ is a Fatou component, $f^{n}(\Omega) \subset A$ for all $n>N$, where $N$ depends on $\Omega$.

Then $J_{r}(f) \neq \varnothing$.

Sketch of Proof B.

Take any point $z \in((\hat{\mathbb{C}} \backslash B) \backslash A) \cap J(f)$ and a neighbourhood $V \subset(\hat{\mathbb{C}} \backslash B) \backslash A$ of $z$. Since periodic points are dense in Julia, then $V$ must contain a periodic point $\zeta$ of the Julia set. Under iteration the point $\zeta$ has to come back to itself infinitely often.

By hypothesis, points on the boundary of any Fatou component must iterate inside $A$ and never leave again. Then points in the Julia which leaves $A$ infinitely often are not in the boundary of a Fatou component, thus $\zeta \in J_{r}(f)$ since it lies in the complement of $A$. Therefore $J_{r}(f) \neq \varnothing$.

\section{REFERENCES}

[1] J. F. Gouyet, "Physics and Fractal Structures," Masson Springer, Paris, New York, 1996.

[2] M. A. Montes de Oca Balderas, G. J. F Sienra Loera and J. E. King Dávalos, "Baker Domains for Period Two for the Family $f_{\lambda, \mu}=\lambda \mathrm{e}^{z}+\frac{\mu}{z}$," International Journal of Bifurcation and Chaos, in Press, 2013.

[3] I. N. Baker, J. Kotus and Y. N. Lü, "Iterates of Meromorphic Functions II: Examples of Wandering Domains," Journal of the London Mathematical Society, Vol. 42, No. 2, 1990, pp. 267-278. http://dx.doi.org/10.1112/jlms/s2-42.2.267

[4] I. N. Baker, J. Kotus and Y. N. Lü, "Iterates of Meromorphic Functions: I," Ergodic Theory and Dynamical Systems, Vol. 11, No. 2, 1991, pp. 241-248.

[5] I. N. Baker, J. Kotus and Y. N. Lü, "Iterates of Mero- 
morphic Functions III. Preperiodic Domains,” Ergodic Theory Dynamical Systems, Vol. 11, 1991, pp. 603-618.

[6] I. N. Baker, J. Kotus and Y. N. Lü, "Iterates of Meromorphic Functions IV. Critically Finite Functions," Results in Mathematics, Vol. 22, No. 2-4, 1992, pp. 651656.

[7] B. Bolsch, "Repulsive Periodic Points of Meromorphic Function," Complex Variables Theory and Application, Vol. 31, No. 1, 1996, pp. 75-79. http://dx.doi.org/10.1080/17476939608814947

[8] A. Bolsch, "Iteration of Meromorphic Functions with Countably Many essential Singularities," Technische Universität Berlin, Berlin, 1997.

[9] A. Bolsch, "Periodic Fatou Components of Meromorphic Functions," Bulletin of the London Mathematical Society, Vol. 31, No. 5, 1999, pp. 543-555. http://dx.doi.org/10.1112/S0024609399005950

[10] W. Abikoff, "Some Remarks on Kleinian Groups," $A n$ nals of Mathematics, Vol. 66, 1971, pp. 1-5.

[11] W. Abikoff, "The Residual Limits Sets of Kleinian Groups,” Acta Mathematica, Vol. 130, No. 1, 1973, pp. 127-144. http://dx.doi.org/10.1007/BF02392264
[12] C. McMullen, “Automorphisms of Rational Maps, Holomorphic Functions and Modulii I,” MSRI Publications 10, Springer Verlag, New York, 1988.

[13] I. N. Baker and P. Domínguez, "Residual Julia Sets," Journal of Analysis, Vol. 8, 2000, pp. 121-137.

[14] J. Y. Qiao, "The Buried Points on the Julia Sets of Rational and Entire Functions," Science in China Series A, Vol. 38, No. 12, 1995, pp. 1409-1419.

[15] P. Domínguez and N. Fagella, "Residual Julia Sets for Rational and Transcendental Functions,” Cambridge University Press, Cambridge, 2008, pp. 138-164. http://dx.doi.org/10.1017/CBO9780511735233.008

[16] P. Domínguez and N. Fagella, "Existence of Herman Rings for Meromorphic Functions,” Complex Variables, Vol. 49, No. 12, 2004, pp. 851-870. http://dx.doi.org/10.1080/02781070412331298589

[17] P. Domínguez, "Residual Julia Sets for Meromorphic Functions with Countably Many Essential Singularities," Journal of Difference Equations and Applications, Vol. 16, No. 5-6, 2010, pp. 519-522. http://dx.doi.org/10.1080/10236190903203879 